\title{
Generating function for $q$-Eulerian polynomials and their decomposition and applications
}

\author{
Mustafa Alkan* and Yilmaz Simsek
}

\section{"Correspondence:}

alkan@akdeniz.edu.tr

Department of Mathematics,

Faculty of Science, Akdeniz

University, Campus, Antalya, 07058,

Turkey

\begin{abstract}
The aim of this paper is to define a generating function for $q$-Eulerian polynomials and numbers attached to any character $\chi$ of the finite cyclic group $G$. We derive many functional equations, $q$-difference equations and partial deferential equations related to these generating functions. By using these equations, we find many properties of $q$-Eulerian polynomials and numbers. Using the generating element of the finite cyclic group $G$ and the generating element of the subgroups of $G$, we show that the generating function with a conductor $f$ can be written as a sum of the generating function with conductors which are less than $f$.

MSC: 05A40; 11B83; 11B68; 11S80

Keywords: Euler numbers; Frobenius-Euler numbers; Frobenius-Euler polynomials; $q$-Frobenius-Euler polynomials; $q$-series; generating function; character $\chi$ of the finite abelian groups $G$
\end{abstract}

\section{Introduction}

The theory of the family of Eulerian polynomials and numbers (Frobenius-Euler polynomials and numbers) has become a very important part of analytic number theory and other sciences, for example, engineering, computer, geometric design and mathematical physics. Euler numbers are related to the Brouwer fixed point theorem and vector fields. Therefore, $q$-Eulerian type numbers may be related to Brouwer fixed point theorem and vector fields [1].

Recently, many different special functions have been used to define and investigate $q$-Eulerian numbers and polynomials, see details [1-39]. Therefore, we construct and investigate various properties of $q$-Eulerian numbers and polynomials, which are related to the many known polynomials and numbers such as Frobenius-Euler polynomials and numbers, Apostol-Euler polynomials and numbers, Euler polynomials and numbers.

Recently in [2-4], the authors defined a relationship between algebra and analysis. In detail, they made a new approximation between the subgroup and monoid presentation and special generating functions (such as Stirling numbers, Array polynomials etc.). In this paper, since our priority aim is to define special numbers and polynomials, it is worth depicting these references as well. Then in this paper, applying any group character $\chi$ of the finite cyclic group $G$ to a special generating function (which has been defined in [5]), we give a generalization of $q$-Eulerian polynomials and numbers (q-Apostol-type Frobenius-Euler 
polynomials and numbers) and investigate their properties and some useful functional equations. Using a generating element of the subgroups of $G$ and a generating element of $G$, we also decompose our generating function attached to the characters of $G$, and so we obtain a new decomposition of $q$-Eulerian numbers and polynomials attached to the characters of a subgroup of $G$ and the Dirichlet character of $G$. This decomposition enables us to compute $q$-Apostol-type Frobenius-Euler polynomials and numbers more easily.

Throughout this paper, we assume that $q \in \mathbb{C}$, the set of complex numbers, with $|q|<1$

$$
[x]=[x: q]= \begin{cases}\frac{1-q^{x}}{1-q} & \text { if } q \neq 1 \\ x & \text { if } q=1\end{cases}
$$

We use the following standard notions.

$\mathbb{N}=\{1,2, \ldots\}, \mathbb{N}_{0}=\{0,1,2, \ldots\}=\mathbb{N} \cup\{0\}$ and also, as usual, $\mathbb{R}$ denotes the set of real numbers, $\mathbb{R}^{+}$denotes the set of positive real numbers and $\mathbb{C}$ denotes the set of complex numbers.

\subsection{Characters of a group $G$}

We recall the definition and some properties of the character of an arbitrary group $(G, \cdot)$ (see [6]).

A non-zero complex-valued function $\chi$ defined on $G$ is called a character of $G$ if for all $a, b \in G$,

$$
\chi(a \cdot b)=\chi(a) \chi(b) .
$$

In particular, if $G$ is a finite group with the identity element $e$, then $\chi(e)=1$ and $\chi(a)$ is a root of unity. In [6, Theorem 6.8], it is proved that a finite abelian group $G$ of order $n$ has exactly $n$ distinct characters.

Let $G$ be the group of reduced residue classes module positive integer $f$. Corresponding to each character $\chi$ of $G$, the Dirichlet character $\chi_{D}$ is defined as follows:

$$
\chi_{D}(n)= \begin{cases}\chi(n) & \text { if } \operatorname{gcd}(n, f)=1 \\ 0 & \text { if } \operatorname{gcd}(n, f)>1\end{cases}
$$

Hence it is easily observed that

$$
\chi(n f+a)=\chi(a)
$$

for all $n, a \in \mathbb{N}$. In this note, $f$ is called the conductor of the character of a group $G$ of reduced residue classes module a positive integer $f$.

\section{$1.2 q$-Eulerian polynomials and numbers}

In [5], Simsek defined and studied some properties of $q$-Apostol type Frobenius-Euler polynomials and numbers.

Definition 1.1 Let $a, b \in \mathbb{R}^{+}(a \neq b)$ and $u \in \mathbb{C} \backslash\{1\}$. 
(i) The $q$-Apostol type Frobenius-Euler numbers

$$
\mathcal{H}_{n}(u ; a, b ; \lambda ; q) \quad(\lambda, q \in \mathbb{C})
$$

are defined by means of the following generating function:

$$
F_{\lambda, q}(t ; u, a, b)=\left(1-\frac{a^{t}}{u}\right) \sum_{n=0}^{\infty}\left(\frac{\lambda}{u}\right)^{n} b^{[n] t}=\sum_{n=0}^{\infty} \mathcal{H}_{n}(u ; a, b ; \lambda ; q) \frac{t^{n}}{n !} .
$$

(ii) The $q$-Apostol type Frobenius-Euler polynomials

$$
\mathcal{H}_{n}(x ; u ; a, b ; \lambda ; q) \quad(\lambda \in \mathbb{C})
$$

are defined by means of the following generating function:

$$
F_{\lambda, q}(x, t ; u, a, b)=\left(1-\frac{a^{t}}{u}\right) \sum_{n=0}^{\infty}\left(\frac{\lambda}{u}\right)^{n} b^{[n+x] t}=\sum_{n=0}^{\infty} \mathcal{H}_{n}(x ; u ; a, b ; \lambda ; q) \frac{t^{n}}{n !},
$$

where

$$
\left|\frac{\lambda}{u} b^{t}\right|<1
$$

\section{$2 q$-Eulerian polynomials and numbers attached to any character}

In this section, we define $q$-Eulerian polynomials and numbers attached to any character of the finite cyclic group $G$. Our new generating functions are related to nonnegative real parameters.

Definition 2.1 Let $a, b \in \mathbb{R}^{+}(a \neq b$ and $a \geq 1), u \in \mathbb{C} \backslash\{1\}, \lambda, q \in \mathbb{C}(|q|<1)$. Let $\chi$ be a character of a finite cyclic group $G$ with the conductor $f$.

(i) The $q$-Eulerian numbers attached to the character $\chi$

$$
\mathcal{H}_{n}, \chi(u ; a, b ; \lambda ; q)
$$

are defined by means of the following generating function:

$$
F_{\lambda, q, \chi}(t, u, a, b)=\left(1-\frac{a^{[f] t}}{u^{f}}\right) \sum_{m=0}^{\infty}\left(\frac{\lambda}{u}\right)^{m} b^{[m] t} \chi(m)=\sum_{n=0}^{\infty} \mathcal{H}_{n, \chi}(u ; a, b ; \lambda ; q) \frac{t^{n}}{n !} .
$$

(ii) The $q$-Eulerian polynomials attached to the character $\chi$

$$
\mathcal{H}_{n, \chi}(x ; u ; a, b ; \lambda ; q)
$$

are defined by means of the following generating function:

$$
F_{\lambda, q, \chi}(t, x, u, a, b)=\left(1-\frac{a^{[f] t}}{u^{f}}\right) \sum_{m=0}^{\infty}\left(\frac{\lambda}{u}\right)^{m} b^{[m+x] t} \chi(m)=\sum_{n=0}^{\infty} \mathcal{H}_{n, \chi}(x ; u ; a, b ; \lambda ; q) \frac{t^{n}}{n !},
$$


where

$$
\left|\frac{\lambda}{u} b^{t}\right|<1
$$

It is observed that

$$
\mathcal{H}_{n, \chi}(0 ; u ; a, b ; \lambda ; q)=\mathcal{H}_{n, \chi}(u ; a, b ; \lambda ; q) .
$$

Upon setting $t=0$ in (3), we compute a $q$-Eulerian number $\mathcal{H}_{0, \chi}(u, a, b, \lambda, q)$ attached to the character $\chi$ as follows:

$$
\mathcal{H}_{0, \chi}(u, a, b, \lambda, q)=\left(\frac{u^{1-f}\left(u^{f}-1\right)}{u-\lambda \chi(1)}\right)
$$

By using the conductor $f$ of the character $\chi$ and combining with $F_{\lambda, q}(t, x, u, a, b)$, we modify Equation (2) and Equation (3), respectively, as follows:

$$
F_{\lambda, q, \chi}(t, u, a, b)=\sum_{i=0}^{f-1}\left(\frac{\lambda \chi(1)}{u}\right)^{i} F_{\lambda f, q^{f}}\left(t[f], \frac{i}{f}, u^{f}, a, b\right)
$$

and

$$
F_{\lambda, q, \chi}(t, x, u, a, b)=\sum_{i=1}^{f}\left(\frac{\lambda \chi(1)}{u}\right)^{i} F_{\lambda f}, q^{f}\left([f] t, \frac{i+x}{f}, u^{f}, a, b\right) .
$$

Therefore, we provide the following relationships between $q$-Eulerian numbers and $q$ Eulerian numbers attached to the character $\chi$.

Theorem 2.2 Let $n \in \mathbb{N}_{0}$. Then we have

(i)

$$
\mathcal{H}_{n, \chi}(u ; a, b ; \lambda, u)=[f]^{n} \sum_{i=0}^{f-1}\left(\frac{\lambda}{u}\right)^{i} \chi(i) \mathcal{H}_{n}\left(\frac{i}{f}, u^{f}, a, b ; \lambda^{f}, q^{f}\right),
$$

(ii)

$$
\mathcal{H}_{n, \chi}(u ; a, b ; \lambda, u)=[f]^{n} \sum_{i=0}^{f-1} \chi(i)\left(\frac{\lambda}{u}\right)^{i} \mathcal{H}_{n}\left(\frac{x+i}{f}, u^{f}, a, b ; \lambda^{f}, q^{f}\right) .
$$

Proof By using (4), we deduce that

$$
\sum_{n=0}^{\infty} \mathcal{H}_{n, \chi}(u ; a, b ; \lambda, u) \frac{t^{n}}{n !}=\sum_{i=0}^{f-1} \chi(i)\left(\frac{\lambda}{u}\right)^{i} \sum_{n=0}^{\infty}[f]^{n} \mathcal{H}_{n}\left(\frac{i}{f}, u^{f}, a, b ; \lambda^{f}, q^{f}\right) \frac{t^{n}}{n !},
$$

which, by comparing the coefficient $\frac{t^{n}}{n !}$ on the both sides of the above equations, yields the first assertion of Theorem 2.2.

The second assertion (ii) is proved with the same argument. 
By Theorem 2.2, we also compute a $q$-Eulerian number attached to the character $\chi$ as follows:

$$
\mathcal{H}_{0, \chi}(u, a, b, \lambda, q)=\sum_{i=0}^{f-1} \chi(i)\left(\frac{\lambda}{u}\right)^{i} \mathcal{H}_{0}\left(\frac{i}{f}, u^{f}, a, b ; \lambda^{f}, q^{f}\right)
$$

Now, we turn our attention to the following generation function defined in [1] since we need this generating function frequently to give some functional equations for a $q$-Eulerian number and polynomials attached to the character $\chi$.

Let $a \geq 1$. The number $Y_{n}(u, a)$ is defined by means of the following generating function:

$$
G(t, u, a)=\frac{1}{a^{t}-u}=\sum_{n=0}^{\infty} Y_{n}(u, a) \frac{t^{n}}{n !}
$$

The polynomials $Y_{n}(x ; u, a)$ are defined by means of the following generating function:

$$
\mathcal{G}(t, x, u, a)=G(t, u, a) a^{x t}=\sum_{n=0}^{\infty} Y_{n}(x ; u, a) \frac{t^{n}}{n !}
$$

Since we need this generating function frequently in this paper, we use the notation

$$
\begin{aligned}
D(k) & =\frac{a^{[f] t}-u^{f}}{a^{[f] q^{k} t}-u^{f}}=\mathcal{G}\left([f] q^{k} t, \frac{1}{q^{k}}, u^{f}, a\right)-u^{f} G\left([f] q^{k} t, u^{f}, a\right) \\
& =\sum_{n=0}^{\infty}[f]^{n} q^{k n}\left(Y_{n}\left(\frac{1}{q^{k}}, u^{f}, a\right)-u^{f} Y_{n}\left(u^{f}, a\right)\right) \frac{t^{n}}{n !},
\end{aligned}
$$

and so it follows that $D_{n}(k)=[f]^{n} q^{n}\left(Y_{n}\left(\frac{1}{q}, u^{f}, a\right)-Y_{n}\left(u^{f}, a\right)\right)$.

By using the following well-known identity:

$$
[n+x]=[x]+q^{x}[n]
$$

in (3), we verify the following functional equation:

$$
F_{\lambda, q, \chi}(t, x+y, u, a, b)=b^{[y] t} D(y) F_{\lambda, q, \chi}\left(q^{y} t, x, u, a, b\right) .
$$

Hence we have the following theorem.

Theorem 2.3 Let $n \in \mathbb{N}_{0}$. Then we have

$$
\begin{aligned}
\mathcal{H}_{n, \chi}(x+y ; u ; a, b ; \lambda ; q)= & \sum_{i=0}^{m} \sum_{j=0}^{i}\left(\begin{array}{c}
m \\
i
\end{array}\right)\left(\begin{array}{l}
i \\
j
\end{array}\right)([y] \ln b)^{i-j} D_{j}(y) q^{(m-i) y} \\
& \times \mathcal{H}_{(m-i) i, \chi}(x, u ; a, b ; \lambda ; q) .
\end{aligned}
$$

Proof By applying the Cauchy product to (7), we deduce that

$$
F_{\lambda, q, \chi}(t, x+y, u, a, b)=\sum_{n=0}^{\infty} \sum_{j=0}^{n}\left(\begin{array}{l}
n \\
j
\end{array}\right)([y] \ln b)^{n-j} D_{j}(y) \frac{t^{n}}{n !} F_{\lambda, q, \chi}\left(t q^{y}, x, u, a, b\right) .
$$


Let $K(n)=\sum_{j=0}^{n}\left(\begin{array}{l}n \\ j\end{array}\right)([y] \ln b)^{n-j} D_{j}(y)$. Then it follows that

$$
\begin{aligned}
F_{\lambda, q, \chi}(t, x+y, u, a, b)= & \sum_{m=0}^{\infty} \sum_{i=0}^{m}\left(\begin{array}{c}
m \\
i
\end{array}\right) K(i) q^{(m-i) y} \mathcal{H}_{m-i, \chi}(x, u ; a, b ; \lambda ; q) \frac{t^{m}}{m !} \\
= & \sum_{m=0}^{\infty} \sum_{i=0}^{m} \sum_{j=0}^{i}\left(\begin{array}{c}
m \\
i
\end{array}\right)\left(\begin{array}{c}
i \\
j
\end{array}\right)([y] \ln b)^{i-j} D_{j}(y) q^{(m-i) y} \\
& \times \mathcal{H}_{(m-i) i, \chi}(x, u ; a, b ; \lambda ; q) \frac{t^{m}}{m !} .
\end{aligned}
$$

By comparing the coefficient of $\frac{t^{m}}{m !}$ on both sides of the above equation, we obtain our desired result.

Upon setting $x=0$ in (7), we get the following functional equation:

$$
\begin{aligned}
F_{\lambda, q, \chi}(t, y, u, a, b) & =b^{[y] t} D(y) F_{\lambda, q, \chi}\left(q^{y} t, 0, u, a, b\right) \\
& =b^{[y] t} D(y) F_{\lambda, q, \chi}\left(q^{y} t, u, a, b\right) .
\end{aligned}
$$

By substituting $x=0$ in Theorem 2.3, the following theorem is easily proved.

Theorem 2.4 Let $n \in \mathbb{N}_{0}$. Then we have

$$
\mathcal{H}_{n, \chi}(y ; u ; a, b ; \lambda ; q)=\sum_{i=0}^{m} \sum_{j=0}^{n-i}\left(\begin{array}{c}
m \\
i
\end{array}\right)\left(\begin{array}{l}
i \\
j
\end{array}\right)([y] \ln b)^{i-j} D_{j}(y) q^{(n-i) y} \mathcal{H}_{(m-i) i, \chi}(u ; a, b ; \lambda ; q) .
$$

So that we obtain a $q$-difference equation for $q$-Eulerian polynomials attached to the character $\chi$, we study the following equations:

$$
\begin{aligned}
F_{\lambda, q, \chi}(t, x, u, a, b) & =\left(1-\frac{a^{[f] t}}{u^{f}}\right)+\frac{\lambda \chi(1)}{u} F_{\lambda, q, \chi}(t, x+1, u, a, b) \\
& =\left(1-\frac{a^{[f] t}}{u^{f}}\right)+\frac{\lambda \chi(1) b^{t}}{u} D(1) F_{\lambda, q, \chi}(q t, x, u, a, b),
\end{aligned}
$$

which, in light of the Cauchy product of the three series $b^{t}, F_{\lambda, q, \chi}(q t, x, u, a, b)$ and

$$
D(1)=\mathcal{G}\left(q[f] t, \frac{1}{q}, u^{f}, a\right)-u^{f} G\left(q[f] t, u^{f}, a\right),
$$

yield the following theorem.

Theorem 2.5 Let $n \in \mathbb{N}_{0}$. Then we have

$$
\mathcal{H}_{0, \chi}(x ; u ; a, b ; \lambda ; q)=1-\frac{([f] \ln a)^{n}}{u^{f}}+\frac{\lambda \chi(1)}{u} C_{0} \mathcal{H}_{0, \chi}(x ; u ; a, b ; \lambda ; q)
$$

and

$$
\mathcal{H}_{n, \chi}(x ; u ; a, b ; \lambda ; q)=-\frac{([f] \ln a)^{n}}{u^{f}}+\frac{\lambda \chi(1)}{u} \sum_{i=0}^{n}\left(\begin{array}{c}
n \\
i
\end{array}\right) C_{n-i} q^{i} \mathcal{H}_{i, \chi}(x ; u ; a, b ; \lambda ; q),
$$


where

$$
C_{n}=\sum_{i=0}^{n}\left(\begin{array}{l}
n \\
i
\end{array}\right)(\ln b)^{n-i}[f]^{i} q^{i}\left(Y_{i}\left(\frac{1}{q}, u^{f}, a\right)-u^{f} Y_{i}(0, u, a)\right)
$$

Now, we turn our attention to studying the derivative of the polynomials

$$
\begin{aligned}
\frac{\partial}{\partial t} F_{\lambda, q, \chi}(x, t, u, a, b)= & \frac{-[f] a^{[f] t} \ln a}{u^{f}} \sum_{n=0}^{\infty}\left(\frac{\lambda}{u}\right)^{n} \chi(n) b^{[n+x] t} \\
& +\left(1-\frac{a^{[f] t}}{u^{f}}\right) \sum_{n=0}^{\infty}\left(\frac{\lambda}{u}\right)^{n} \chi(n)[n+x] b^{[n+x] t} \ln b .
\end{aligned}
$$

By

$$
\sum_{i=0}^{\infty} y^{n}[n]=\frac{1}{(1-y)(1-q)}-\frac{y}{(1-q y)(1-q)}
$$

and substituting $t=0$ in (8), we get that

$$
\begin{aligned}
\mathcal{H}_{1, \chi}(x ; u ; a, b ; \lambda ; q)= & \frac{-[f] \ln a}{u^{f}-u^{f-1} \lambda \chi(1)}+\left(\frac{u^{f}-1}{u^{f}}\right)\left(\frac{u}{u-\lambda \chi(1)}\right)[x] \ln b \\
& +\left(\frac{u^{f}-1}{u^{f}(1-q)}\right) q^{x}\left(\frac{u}{u-\lambda \chi(1)}+\frac{u}{u-q \lambda \chi(1)}\right) .
\end{aligned}
$$

Hence, using the induction method, we arrive at the following result.

Theorem 2.6 Let $n \in \mathbb{N}_{0}$. Then we have

$$
\mathcal{H}_{n, \chi}(x ; u ; a, b ; \lambda ; q) \frac{t^{n}}{n !}=\left.\frac{\partial^{n}}{\partial t^{n}} F_{\lambda, q, \chi}(x, t, u, a, b)\right|_{t=0} .
$$

Now we give a generalization of the Raabe formula by the following theorem.

Theorem 2.7 Let $c, n \in \mathbb{N}_{0}$. Then we have

$$
\begin{aligned}
\mathcal{H}_{n, \chi}(x ; u ; a, b ; \lambda ; q)= & \sum_{n=0}^{c-1} \sum_{j=0}^{n}\left(\begin{array}{l}
n \\
j
\end{array}\right) \frac{\lambda \chi(1)}{u} \mathcal{R}_{j}(c)[c]^{n-j} \\
& \times \mathcal{H}_{n-j, \chi_{c}}\left(\frac{x+l}{c} ; u^{c} ; a, b ; \lambda^{c} ; q^{c}\right),
\end{aligned}
$$

where

$$
\mathcal{R}_{j}(c)=\left[c f_{c}\right]^{j}\left(Y_{n}\left(\frac{[f]}{\left[c f_{c}\right]} ; u^{f}, a\right)-u^{f} Y_{n}\left(u^{f}, a\right)\right)
$$

and

$$
\chi_{c}(x)=\chi(c x)
$$


Proof We start the proof with defining the character

$$
\chi_{c}(x)=\chi(c x)
$$

with the conductor $f_{c}=\frac{f}{\operatorname{gcd}(f, c)}$. On the other hand, we derive that

$$
\begin{aligned}
\left(\frac{u^{f}-a^{[f] t}}{u^{f}}\right) & =\frac{u^{f}-a^{[f] t}}{u^{f}-a^{\left[f_{c}\right]_{q^{c}}[c] t}} \frac{u^{f}-a^{\left[f_{c}\right]_{q} c[c] t}}{u^{f}} \\
& =\mathcal{R}(c) \frac{u^{f}-a^{\left[f_{c}\right]_{q}[c] t}}{u^{f}}
\end{aligned}
$$

where

$$
\mathcal{R}(c)=\frac{a^{[f] t}-u^{f}}{a^{\left[c f_{c}\right] t}-u^{f}} .
$$

Then by using (6), it follows that

$$
\begin{aligned}
\mathcal{R}(c) & =\mathcal{G}\left(\left[c f_{c}\right] t, \frac{[f]}{\left[c f_{c}\right]}, u^{f}, a\right)-u^{f} \mathcal{G}\left(\left[c f_{c}\right] t, 0, u^{f}, a\right) \\
& =\sum_{n=0}^{\infty}\left[c f_{c}\right]^{n}\left(Y_{n}\left(\frac{[f]}{\left[c f_{c}\right]} ; u^{f}, a\right)-u^{f} Y_{n}\left(u^{f}, a\right)\right) \frac{t^{n}}{n !} \\
& =\sum_{n=0}^{\infty} \mathcal{R}_{n}(c) \frac{t^{n}}{n !} .
\end{aligned}
$$

Now, we are ready to prove our result.

$$
\begin{aligned}
& \sum_{n=0}^{\infty} \mathcal{H}_{n, \chi}(x ; u ; a, b ; \lambda ; q) \frac{t^{n}}{n !} \\
& \quad=\left(1-\frac{a^{[f] t}}{u^{f}}\right) \sum_{m=0}^{\infty} \sum_{l=0}^{c-1}\left(\frac{\lambda}{u}\right)^{l+m c} b^{[l+m c+x] t} \chi(l+m c) \\
& =\left(1-\frac{a^{[f] t}}{u^{f}}\right) \sum_{l=0}^{c-1}\left(\frac{\lambda \chi(1)}{u}\right) \sum_{m=0}^{l}\left(\frac{\lambda^{c}}{u^{c}}\right) b^{\left[m+\frac{l+x}{c}\right]_{q^{c}}[c] t} \chi(c m) \\
& \quad=\sum_{l=0}^{c-1}\left(\frac{\lambda \chi(1)}{u}\right)^{l} \sum_{n=0}^{\infty} \mathcal{R}_{n}(c) \frac{t^{n}}{n !} \sum_{n=0}^{\infty}[c]^{n} \mathcal{H}_{n, \chi_{c}}\left(\frac{x+l}{c} ; u^{c} ; a, b ; \lambda^{c} ; q^{c}\right) \frac{t^{n}}{n !} \\
& =\sum_{n=0}^{\infty} \sum_{l=0}^{c-1}\left(\frac{\lambda \chi(1)}{u}\right)^{l} \sum_{j=0}^{n}\left(\begin{array}{l}
n \\
j
\end{array}\right) \mathcal{R}_{j}(c)[c]^{n-j} \mathcal{H}_{n-j, \chi_{c}}\left(\frac{x+l}{c} ; u^{c} ; a, b ; \lambda^{c} ; q^{c}\right) \frac{t^{n}}{n !} .
\end{aligned}
$$

Hence, comparing the coefficient of $\frac{t^{n}}{n !}$ on both sides yields the assertion of this theorem.

\section{Decomposition of the generating function}

In this section, using the generating element of the finite cyclic group $G$ and the generating element of the subgroups of $G$, we show that the generating function with a conductor $f$ can be written as a sum of the generating function with conductors which are less than $f$. 
In this section, we use the following notations otherwise stated:

(i) $x \mathbb{N}_{0}=\{0, x, 2 x, 3 x, 4 x, \ldots\}$,

(ii) $p_{i}$ denotes the integer such that $\operatorname{gcd}\left(p_{i}, p_{j}\right)=1$ for all $i, j \in\{1, \ldots, n\}$,

(iii) $S=\bigcup_{i=1}^{n}\left(p_{i} \mathbb{N}_{0}\right)$,

(iv) $S_{0}=\left\{p_{1}, \ldots, p_{n}\right\}$,

(v) $S_{i}=\left\{\operatorname{lcd}(a, b): a, b \in S_{i-1}\right\}$.

We start to recall the fact that

$$
x \mathbb{N}_{0} \cap y \mathbb{N}_{0}=\operatorname{lcd}(x, y) \mathbb{N}_{0}
$$

for positive integers $x$ and $y$. In particular, we get that

$$
x^{k} \mathbb{N}_{0} \cap y^{t} \mathbb{N}_{0}=x y \mathbb{N}_{0}
$$

whenever $x$ and $y$ are distinct prime numbers.

Theorem 3.1 With the above notations, we get

$$
\sum_{i \in S} F(i)=\sum_{j=0}^{n-1}(-1)^{j} \sum_{t \in S_{j}} \sum_{i \in t \mathbb{N}_{0}} F(i) .
$$

Proof We start to recall the fact that for sets $A, B$,

$$
\sum_{i \in A \cup B} F(i)=\sum_{i \in A} F(i)+\sum_{i \in B} F(i)-\sum_{i \in A \cap B} F(i) .
$$

Then by using De-Morgan's law of sets, we get the following equality:

$$
\begin{aligned}
\sum_{i \in\left(p_{1} \mathbb{N}_{0}\right) \cup\left(\bigcup_{i=2}^{n}\left(p_{i} \mathbb{N}_{0}\right)\right)} F(i) & =\sum_{i \in\left(p_{1} \mathbb{N}_{0}\right)} F(i)+\sum_{i \in\left(\bigcup_{i=2}^{n}\left(p_{i} \mathbb{N}_{0}\right)\right)} F(i)-\sum_{i \in\left(p_{1} \mathbb{N}_{0}\right) \cap\left(\bigcup_{i=2}^{n}\left(p_{i} \mathbb{N}_{0}\right)\right)} F(i) \\
& =\sum_{i \in\left(p_{1} \mathbb{N}_{0}\right)} F(i)+\sum_{i \in\left(\bigcup_{i=2}^{n}\left(p_{i} \mathbb{N}_{0}\right)\right)} F(i)-\sum_{i \in\left(\bigcup_{i=2}^{n} \operatorname{lcd}\left(p_{1}, p_{i}\right) \mathbb{N}_{0}\right)} F(i) .
\end{aligned}
$$

Now we use induction on $n$.

If $n=2$, then $S_{0}=\left\{p_{1}, p_{2}\right\}$ and $S_{1}=\left\{\operatorname{lcd}\left(p_{1}, p_{2}\right)\right\}$. We have

$$
\begin{aligned}
\sum_{i \in\left(\bigcup_{i=1}^{2}\left(p_{i} \mathbb{N}_{0}\right)\right)} F(i) & =\sum_{i \in\left(p_{1} \mathbb{N}_{0}\right)} F(i)+\sum_{i \in\left(p_{2} \mathbb{N}_{0}\right)} F(i)-\sum_{i \in \operatorname{lcd}\left(p_{1}, p_{2}\right) \mathbb{N}_{0}} F(i) \\
& =\sum_{j=0}^{1}(-1)^{j} \sum_{t \in S_{j}} \sum_{i \in t \mathbb{N}_{0}} F(i) .
\end{aligned}
$$

We construct the following sets:

$$
\begin{aligned}
& S=\bigcup_{i=1}^{n}\left(p_{i} \mathbb{N}_{0}\right), \\
& S^{\prime}=\bigcup_{i=2}^{n}\left(p_{i} \mathbb{N}_{0}\right)
\end{aligned}
$$


and

$$
S=\left(p_{1} \mathbb{N}_{0}\right) \cup S^{\prime}
$$

By using De-Morgan's law of sets, we verify the following equalities:

$$
\begin{aligned}
& S^{\prime \prime}=\left(p_{1} \mathbb{N}_{0}\right) \cap S^{\prime}=\bigcup_{i=2}^{n}\left(\operatorname{lcd}\left(p_{1}, p_{i}\right) \mathbb{N}_{0}\right), \\
& S_{0}=S_{0}^{\prime} \cup\left\{p_{1}\right\}, \\
& S_{1}=\left\{\operatorname{lcd}(a, b): a, b \in S_{0}\right\}=S_{1}^{\prime} \cup S_{0}^{\prime \prime}, \\
& S_{2}=\left\{\operatorname{lcd}(a, b): a, b \in S_{1}\right\}=S_{2}^{\prime} \cup S_{1}^{\prime \prime}, \\
& S_{i}=\left\{\operatorname{lcd}(a, b): a, b \in S_{i-1}\right\}=S_{i}^{\prime} \cup S_{i-1}^{\prime \prime}
\end{aligned}
$$

and for all $i \geq 1$, it is clear that $S_{i}^{\prime} \cap S_{i-1}^{\prime \prime}=\oslash$.

Now assume that it is true for the set with $n-1$ elements. Hence, the hypothesis for the sets $S^{\prime \prime}$ and $S^{\prime}$ is true, and we get that

$$
\sum_{i \in S^{\prime \prime}} F(i)=\sum_{j=0}^{n-2}(-1)^{j} \sum_{t \in S_{j}^{\prime \prime}} \sum_{i \in t \mathbb{N}_{0}} F(i)
$$

and

$$
\sum_{i \in S^{\prime}} F(i)=\sum_{j=0}^{n-2}(-1)^{j} \sum_{t \in S_{j}^{\prime}} \sum_{i \in t \mathbb{N}_{0}} F(i) .
$$

Hence it follows

$$
\begin{aligned}
\sum_{i \in S} F(i) & =\sum_{i \in p_{1} \mathbb{N}_{0}} F(i)+\sum_{i \in S^{\prime}} F(i)-\sum_{i \in S^{\prime \prime}} F(i) \\
& =\sum_{i \in p_{1} \mathbb{N}_{0}} F(i)+\sum_{j=0}^{n-2}(-1)^{j} \sum_{t \in S_{j}^{\prime}} \sum_{i \in t \mathbb{N}_{0}} F(i)-\sum_{j=0}^{n-2}(-1)^{j} \sum_{t \in S_{j}^{\prime \prime}} \sum_{i \in t \mathbb{N}_{0}} F(i) \\
& =\sum_{t \in S_{0}} \sum_{i \in t \mathbb{N}_{0}} F(i)-\sum_{t \in S_{1}^{\prime} \cup S_{0}^{\prime \prime}} \sum_{i \in t \mathbb{N}_{0}} F(i)+\sum_{j=2}^{n-2}(-1)^{j} \sum_{t \in S_{j}^{\prime} \cup S_{j-1}^{\prime \prime}} \sum_{i \in t \mathbb{N}_{0}} F(i) \\
& =\sum_{j=0}^{n-1}(-1)^{j} \sum_{t \in S_{j}} \sum_{i \in t \mathbb{N}_{0}} F(i) .
\end{aligned}
$$

Example 3.2 Let $p_{1}, p_{2}, p_{3}$ be distinct prime integers. Then we have

$$
\begin{aligned}
\sum_{i \in\left(\bigcup_{i=1}^{3}\left(p_{i} \mathbb{N}_{0}\right)\right)} F(i)= & \sum_{i \in\left(p_{1} \mathbb{N}_{0}\right)} F(i)+\sum_{i \in\left(p_{2} \mathbb{N}_{0}\right)} F(i)+\sum_{i \in\left(p_{3} \mathbb{N}_{0}\right)} F(i)-\sum_{i \in\left(p_{2} p_{3} \mathbb{N}_{0}\right)} F(i) \\
& -\sum_{i \in\left(p_{1} p_{2} \mathbb{N}_{0}\right)} F(i)-\sum_{i \in\left(p_{1} p_{3} \mathbb{N}_{0}\right)} F(i)+\sum_{i \in\left(p_{1} p_{2} p_{3} \mathbb{N}_{0}\right)} F(i) .
\end{aligned}
$$


Lemma 3.3 Let $p_{i}$ be a prime number for all $i$ and

$$
C=\left\{l \in \mathbb{N}_{0}: \operatorname{gcd}(f, l)=1\right\} .
$$

Then $\mathbb{N}_{0}=C \cup\left(\bigcup_{i=1}^{n}\left(p_{i} \mathbb{N}_{0}\right)\right)$ and $C \cap\left(\bigcup_{i=1}^{n}\left(p_{i} \mathbb{N}_{0}\right)\right)$ is an empty set.

Proof By using the fact $(l, f)=1$ if and only if $\left(l, \prod_{i=1}^{n} p_{i}\right)=1$ if and only if $\left(l, p_{i}\right)=1$ for all $i \in\{1, \ldots, n\}$ for $n \in \mathbb{N}_{0}$, we get that

$$
\begin{aligned}
C & =\left\{l \in \mathbb{N}_{0}: \operatorname{gcd}(f, l)=1\right\} \\
& =\left\{l \in \mathbb{N}_{0}: \text { for all } i \in\{1, \ldots, n\}, \operatorname{gcd}\left(p_{i}, l\right)=1\right\} .
\end{aligned}
$$

Let $x \notin \bigcup_{i=1}^{n}\left(p_{i} \mathbb{N}_{0}\right)$. Then we get that $\operatorname{gcd}\left(x, p_{i}\right)=1$ for all $i$ and so $x \in C$.

If $x \notin C$, then there is $i \in\{1, \ldots, n\}$ such that $\operatorname{gcd}\left(x, p_{i}\right)$ is $p_{i}$. Therefore

$$
\mathbb{N}_{0}=C \cup\left(\bigcup_{i=1}^{n}\left(p_{i} \mathbb{N}_{0}\right)\right) .
$$

Let $x \in C \cap\left(\bigcup_{i=1}^{n}\left(p_{i} \mathbb{N}_{0}\right)\right)$. Then there is $i \in\{1, \ldots, n\}$ such that $x \in p_{i} \mathbb{N}_{0}$. This means that $\operatorname{gcd}\left(x, p_{i}\right)=p_{i}$ and so $x \notin C$, a contradiction. Thus the proof is completed.

By using the Lemma 3.3, we have one of the main results in this section.

Theorem 3.4 With the above notations, we get that

$$
\sum_{i \in \mathbb{N}_{0}} F(i)=\sum_{i \in C} F(i)+\sum_{i \in\left(\bigcup_{j=1}^{n}\left(p_{j} \mathbb{N}_{0}\right)\right)} F(i) .
$$

As stated before, to decompose the generating function of $q$-Eulerian polynomials attached to the character $\chi$, now we need to compute the following relation:

$$
\sum_{n \in(d Z)}\left(\frac{\lambda}{u}\right)^{n} b^{[n] t} \chi(n)
$$

where

$$
f_{d}=\frac{f}{d} \in \mathbb{N}_{0} .
$$

Also, we define the character $\chi_{g}$ with the conductor $h$ such as

$$
\chi_{g}(i):=\chi(g i)
$$

where $g, h \in \mathbb{N}_{0}$ and $\operatorname{gcd}(g, h)=1$. Then

$$
\begin{aligned}
\sum_{n \in(d Z)}\left(\frac{\lambda}{u}\right)^{n} b^{[n] t} \chi(n) & =\sum_{n \in \mathbb{N}_{0}}\left(\frac{\lambda}{u}\right)^{d n} b^{[d n] t} \chi(d n) \\
& =\sum_{n \in \mathbb{N}_{0}} \sum_{i=0}^{f_{d}-1}\left(\frac{\lambda}{u}\right)^{d\left(f_{d} n+i\right)} b^{\left[d\left(f_{d} n+i\right)\right] t} \chi\left(d\left(f_{d} n+i\right)\right)
\end{aligned}
$$




$$
\begin{aligned}
& =\sum_{n \in \mathbb{N}_{0}} \sum_{i=0}^{f_{d}-1}\left(\frac{\lambda^{d}}{u^{d}}\right)^{f_{d} n+i} b^{t[d]\left[f_{d} n+i\right]} q^{d} \chi_{d}(i) \\
& =\sum_{i \in \mathbb{N}_{0}}\left(\frac{\lambda^{d}}{u^{d}}\right)^{i} b^{t[d][i]} q^{d} \chi_{d}(i) .
\end{aligned}
$$

Hence, we combine the above equation with the generating function

$$
\begin{aligned}
\left(1-\frac{a^{[f] t}}{u^{f}}\right) \sum_{n \in(d Z)}\left(\frac{\lambda}{u}\right)^{n} b^{[n] t} \chi(n) & =\left(1-\frac{a^{\left[d f_{d}\right] t}}{u^{d f}}\right) \sum_{i \in \mathbb{N}_{0}}\left(\frac{\lambda}{u}\right)^{i} b^{[i] q^{d} t} \chi_{d}(i) \\
& =\left(1-\frac{a^{[d]\left[f_{d}\right]_{q^{d}} t}}{\left(u^{d}\right)^{f_{d}}}\right) \sum_{i \in \mathbb{N}_{0}}\left(\frac{\lambda}{u}\right)^{i} b_{d}^{[i]^{d} t} \chi_{d}(i) \\
& =F_{\lambda^{d}, q^{d}, \chi_{d}}\left(t, u^{d}, a^{[d]}, b^{[d]}\right) .
\end{aligned}
$$

Now we are ready to state the main result without the proof in this section.

Theorem 3.5 Let $f=\prod_{i=1}^{n} p_{i}^{t_{t}}$, where $p_{i}$ is a prime integer. Then

$$
F_{\lambda, q, \chi}(t, u, a, b)=\sum_{j=0}^{n-1}(-1)^{j} \sum_{t \in S_{j}} \sum_{i \in t Z} F_{\lambda^{i}, q^{i}, \chi_{i}}\left(t, u^{i}, a^{[i]}, b^{[i]}\right)+F_{\lambda, q, \chi_{D}}(t, u, a, b),
$$

where $\chi_{D}$ is a Dirichlet character corresponding to the character $\chi$.

Example 3.6 Let $f=p_{1}^{t_{1}} p_{2}^{t_{2}} p_{3}^{t_{3}}$, where $p_{i}$ is a prime integer. Then

$$
\begin{aligned}
& F_{\lambda, q, \chi}(t, u, a, b)=F_{\lambda, q, \chi_{D}}(t, u, a, b)+\sum_{i=1}^{3} F_{\lambda p_{i}, q^{p_{i}, \chi_{p_{i}}}}\left(t, u^{p_{i}}, a^{\left[p_{i}\right]}, b^{\frac{\left[p_{i}\right]}{\left[p_{i}\right] q^{p_{i}}}}\right)
\end{aligned}
$$

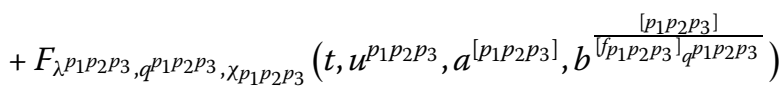

$$
\begin{aligned}
& -\sum_{i=1}^{3} F_{\lambda} p_{1} p_{i}, q_{1}^{p_{1} p_{i}, \chi_{p_{1} p_{i}}}\left(t, u^{p_{1} p_{i}}, a^{\left[p_{1} p_{i}\right]}, b^{\frac{\left[p_{1} p_{i}\right]}{\left[F_{1} p_{i} q_{q} p_{1} p_{i}\right.}}\right)
\end{aligned}
$$

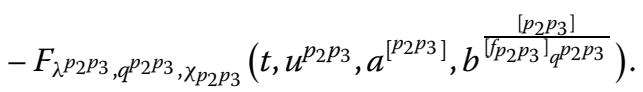

Competing interests

The authors declare that they have no competing interests.

\section{Authors' contributions}

Both authors completed the paper together. Both authors read and approved the final manuscript.

\section{Acknowledgements}

Dedicated to Professor Hari M Srivastava.

All authors are partially supported by Research Project Offices Akdeniz Universities. The author would like to thank to all referees for their valuable comments and also for suggesting references [7-14]. 


\section{References}

1. Simsek, Y: Generating functions for generalized Stirling type numbers, array type polynomials, Eulerian type polynomials and their applications. Fixed Point Theory Appl. 2013, 87 (2013). doi:10.1186/1687-1812-2013-87

2. Cangül, IN, Cevik, AS, Simsek, Y: Analysis approach to finite monoids. Fixed Point Theory Appl. 2013, 15 (2013). doi:10.1186/1687-1812-2013-15

3. Cevik, AS, Cangül, IN, Simsek, Y: A new approach to connect algebra with analysis: relationship and applications between presentations and generating functions. Bound. Value Probl. 2013, 51 (2013). doi:10.1186/1687-2770-2013-51

4. Cevik, AS, Das, KC, Simsek, Y, Cangül, IN: Some array polynomials over special monoids presentations. Fixed Point Theory Appl. 2013, 44 (2013). doi:10.1186/1687-1812-2013-44

5. Simsek, Y: Generating functions for q-Apostol type Frobenius-Euler numbers and polynomials. Axioms 1, 395-403 (2012). doi:10.3390/axioms1030395

6. Apostol, TM: Introduction to Analytic Number Theory. Springer, New York (1976)

7. Luo, QM, Srivastava, HM: Some relationships between the Apostol-Bernoulli and Apostol-Euler polynomials. Comput. Math. Appl. 10, 631-642 (2005)

8. Luo, QM, Srivastava, HM: Some generalizations of the Apostol-Bernoulli and Apostol-Euler polynomials. J. Math. Anal. Appl. 308, 290-302 (2005)

9. Luo, QM: Apostol-Euler polynomials of higher order and Gaussian hypergeometric functions. Taiwan. J. Math. 10(4), 917-925 (2006)

10. Luo, QM, Srivastava, HM: Some relationships between Apostol-Bernoulli and Apostol-Euler polynomials. Comput. Math. Appl. 51, 631-642 (2006)

11. Luo, QM, Srivastava, HM: q-extensions of some relationships between the Apostol-Bernoulli and Apostol-Euler polynomials. Taiwan. J. Math. 15, 241-257 (2011)

12. Luo, QM: Some results for the q-Bernoulli and q-Euler polynomials. J. Math. Anal. Appl. 363, 7-18 (2010)

13. Luo, QM: An explicit relationships between the generalized Apostol-Bernoulli and Apostol-Euler polynomials associated with $\boldsymbol{\lambda}$-Stirling numbers of the second kind. Houst. J. Math. 36, 1159-1171 (2010)

14. Luo, QM: Some formulas for the Apostol-Euler polynomials associated with Hurwitz zeta function at rational arguments. Appl. Anal. Discrete Math. 3, 336-346 (2009)

15. Choi, J, Anderson, PJ, Srivastava, HM: Carlitz's q-Bernoulli and q-Euler numbers and polynomials and a class of generalized q-Hurwitz zeta functions. Appl. Math. Comput. 215, 1185-1208 (2009)

16. Kim, T, Jang, CL, Park, HK: A note on q-Euler and Genocchi numbers. Proc. Jpn. Acad. 77, 139-141 (2001)

17. Simsek, Y, Bayad, A, Lokesha, V: q-Bernstein polynomials related to $q$-Frobenius-Euler polynomials, I-functions, and $q$-Stirling numbers. Math. Methods Appl. Sci. 35, 877-884 (2012)

18. Srivastava, HM, Choi, J: Zeta and q-Zeta Functions and Associated Series and Integrals. Elsevier, Amsterdam (2012)

19. Srivastava, HM: Some generalizations and basic (or q-) extensions of the Bernoulli, Euler and Genocchi polynomials. Appl. Math. Inf. Sci. 5, 390-444 (2011)

20. Kim, T: A note on some formulae for the q-Euler numbers and polynomials. Proc. Jangjeon Math. Soc. 9, 227-232 (2006). arXiv:math/0608649

21. Kim, T: The modified q-Euler numbers and polynomials. Adv. Stud. Contemp. Math. 16, 161-170 (2008) arXiv:math/0702523

22. Kim, T: A note on the alternating sums of powers of consecutive $q$-integers. Adv. Stud. Contemp. Math. 11, 137-140 (2005). arXiv:math/0604227v1 [math.NT]

23. Carlitz, L: q-Bernoulli and Eulerian numbers. Trans. Am. Math. Soc. 76, 332-350 (1954)

24. Choi, J, Anderson, PJ, Srivastava, HM: Some $q$-extensions of the Apostol-Bernoulli and the Apostol-Euler polynomials of order $n$, and the multiple Hurwitz zeta function. Appl. Math. Comput. 199, 723-737 (2008)

25. Kim, T: On a $q$-analogue of the $p$-adic log gamma functions and related integrals. J. Number Theory 76, 320-329 (1999)

26. Jang, LC, Kim, T: q-analogue of Euler-Barnes' numbers and polynomials. Bull. Korean Math. Soc. 42, $491-499$ (2005)

27. Kim, T: On the q-extension of Euler and Genocchi numbers. J. Math. Anal. Appl. 326, 1458-1465 (2007)

28. Kim, T, Jang, LC, Rim, SH, Pak, HK: On the twisted q-zeta functions and q-Bernoulli polynomials. Far East J. Appl. Math. 13, 13-21 (2003)

29. Kim, T, Rim, SH: A new Changhee $q$-Euler numbers and polynomials associated with $p$-adic $q$-integral. Comput. Math Appl. 54, 484-489 (2007)

30. Ozden, $\mathrm{H}$, Simsek, Y: A new extension of $q$-Euler numbers and polynomials related to their interpolation functions. Appl. Math. Lett. 21, 934-939 (2008)

31. Ozden, H, Simsek, Y, Srivastava, HM: A unified presentation of the generating functions of the generalized Bernoulli, Euler and Genocchi polynomials. Comput. Math. Appl. 60, 2779-2787 (2010)

32. Schempp, W: Euler-Frobenius polynomials. In: Numer. Methods Approx. Theory, vol. 7 (Oberwolfach, 1983). Internat. Schriftenreihe Numer. Math., vol. 67, pp. 131-138 (1984)

33. Shiratani, K: On Euler numbers. Mem. Fac. Sci. Kyushu Univ. 27, 1-5 (1975)

34. Simsek, Y: Twisted $(h, q)$-Bernoulli numbers and polynomials related to twisted $(h, q)$-zeta function and $L$-function J. Math. Anal. Appl. 324, 790-804 (2006)

35. Simsek, Y: $q$-analogue of the twisted $/$-series and $q$-twisted Euler numbers. J. Number Theory 110, 267-278 (2005)

36. Simsek, Y: On $p$-adic twisted $q$-L-functions related to generalized twisted Bernoulli numbers. Russ. J. Math. Phys. 13, 327-339 (2006)

37. Simsek, Y, Kim, T, Park, DW, Ro, YS, Jang, LC, Rim, SH: An explicit formula for the multiple Frobenius-Euler numbers and polynomials. JP J. Algebra Number Theory Appl. 4, 519-529 (2004)

38. Srivastava, HM, Kim, T, Simsek, Y: q-Bernoulli numbers and polynomials associated with multiple $q$-zeta functions and basic L-series. Russ. J. Math. Phys. 12, 241-268 (2005)

39. Tsumura, $\mathrm{H}$ : A note on $q$-analogues of the Dirichlet series and q-Bernoulli numbers. J. Number Theory 39, 251-256 (1991) 
doi:10.1186/1687-1812-2013-72

Cite this article as: Alkan and Simsek: Generating function for $q$-Eulerian polynomials and their decomposition and applications. Fixed Point Theory and Applications 2013 2013:72.

Submit your manuscript to a SpringerOpen ${ }^{\circ}$ journal and benefit from:

- Convenient online submission

- Rigorous peer review

- Immediate publication on acceptance

Open access: articles freely available online

- High visibility within the field

- Retaining the copyright to your article

Submit your next manuscript at $\gg$ springeropen.com 